\title{
Special issue dedicated to the 2011 Humboldt-Princeton Workshop on mathematical finance
}

\author{
Ulrich Horst • Ronnie Sircar
}

Published online: 16 March 2013

(C) Springer-Verlag Berlin Heidelberg 2013

The 3rd Humboldt-Princeton Workshop was held in Berlin on October 28-29th, 2011, bringing together Mathematicians, Economists and Statisticians for a lively and stimulating meeting. The four papers in this special issue reflect some of the diversity and interesting problems presented and discussed at the workshop.

The first paper, by Rene Carmona and Francois Delarue, analyzes the connections between Mean Field Games and the McKean-Vlasov equation, motivated by application to cap-andtrade models for greenhouse gas emissions, and using techniques of backward stochastic differential equations.

The second paper, by Rene Carmona, Michael Coulon and Daniel Schwarz, is on the topic of electricity markets and understanding how their dynamics arises from the order in which energy is supplied from different fuel sources. The structural model developed here is used to provide tractable formulas for electricity forward contracts and spread options.

The third paper, by Zorana Grbac and Antonis Papapantoleon, constructs an analytically tractable gramework for modeling the LIBOR interest rates while incorporating default risk. This is based on the theory of affine processes, and the authors highlight the application to valuing CDS spreads and counterparty risk.

The final paper, by Andreas Hamel, Birgit Rudloff and Mihaela Yankova, studies construction and computation of a family of set-valued risk measures, that can be used to quantify the risk of portfolios that contain assets in different currencies, for instance. In this paper, the mathematics and numerical issues are illustrated with the set-valued average value at risk measure.

The papers cover a wide range of topics in Mathematical Finance and were a pleasure for us, as guest editors, to read. We thank the participants of the Humboldt-Princeton Workshop,

U. Horst ( $\varangle)$

Humboldt University, Berlin, Germany

e-mail: horst@math.hu-berlin.de

R. Sircar

Princeton University, Princeton, NJ, USA

e-mail: sircar@princeton.edu 
as well as our colleagues Jianqing Fan (Princeton) and Wolfgang Haerdle (Humboldt) who originated and co-organized this series.

Ulrich Horst (Humboldt University)

Ronnie Sircar (Princeton University) 